\title{
EP-148
}

\section{Postoperative outcomes after laparoscopic cholecystectomy in elderly}

\author{
Daejoon PARK ${ }^{1}$, Huisong LEE', Seog Ki MIN², Hyun Kook LEE*,2 \\ 'Department of General Surgery, Ewha Womans University Mokdong Hospital, Seoul, Korea \\ ${ }^{2}$ Department of General Surgery, Ewha Womans University Seoul Hospital, Seoul, Korea
}

Introduction: Gallstone disease is common morbidity in elderly (age $\geq 65$ years old). Laparoscopic cholecystectomy is increasingly performed in an ever elderly. However, there are no clarified standards for treatment for asymptomatic cholelithiasis in elderly. The purpose of this study was to compare postoperative outcomes of elderly with those of younger populations and to suggest an appropriate age for preventive cholecystectomy before the risk increases.

Methods: In a prospective Korea Cholecystectomy Quality Improvement Program cohort including 18 institutions from October 2016 to March 2017, 2,610 patients who underwent laparoscopic cholecystectomy for benign gallbladder disease were enrolled. Various characteristics, preoperative findings, operative findings were compared between two groups (age $<65$ years old versus age $\geq 65$ years old).

Results: Increasing age was significantly associated with increased rates of overall complications (odds ratio [OR]: 1.79, CI 95\%: 1.452.20), major complication (OR: 1.64, CI 95\%: 1.35-2.08), risk of conversion to open cholecystectomy (OR: 2.00, CI 95\%: 1.76-2.28), and was significantly associated with increased length of stay (MD: 2.56 days, CI 95\%: 1.24-3.42). Differences in mortality and bile duct injury were non-significant. And most appropriate age that postoperative risk increases is 71 years old (AUC 0.74 ).

Conclusions: Postoperative outcomes such as overall and major complications appear to be significantly higher in elderly. Even with asymptomatic cholelithiasis, if the size is more than $1 \mathrm{~cm}$, it is better to recommend surgery before the patient's age reaches 71 . 